\title{
Nonlinear Dimensionality Reduction Using Circuit Models
}

\author{
Fredrik Andersson and Jens Nilsson \\ Centre for Mathematical Sciences, \\ Lund University/LTH, \\ P.O. Box 118, S-22100 Lund, Sweden \\ \{fa, jensn\}@maths.1th.se
}

\begin{abstract}
The problem addressed in nonlinear dimensionality reduction, is to find lower dimensional configurations of high dimensional data, thereby revealing underlying structure. One popular method in this regard is the Isomap algorithm, where local information is used to find approximate geodesic distances. From such distance estimations, lower dimensional representations, accurate on a global scale, are obtained by multidimensional scaling. The property of global approximation sets Isomap in contrast to many competing methods, which approximate only locally.

A serious drawback of Isomap is that it is topologically instable, i.e., that incorrectly chosen algorithm parameters or perturbations of data may abruptly alter the resulting configurations. To handle this problem, we propose new methods for more robust approximation of the geodesic distances. This is done using a viewpoint of electric circuits. The robustness is validated by experiments. By such an approach we achieve both the stability of local methods and the global approximation property of global methods.
\end{abstract}

\section{Introduction}

The analysis of data sampled from manifolds embedded in higher dimensional spaces, is often referred to as manifold learning or nonlinear dimensionality reduction. Such situations arise frequently across diverse disciplines of science such as image analysis, signal processing, psychology and biology. The recent years have brought a growing interest in the development of methods for cases where data are sampled from curved manifolds and where standard, well-established methods like Principal Component Analysis (PCA) [10] and Multidimensional Scaling (MDS) [6] do not perform satisfactory. Locally Linear Embedding [12], Laplacian Eigenmaps [3] and Hessian Eigenmaps [8] represent recent efforts to address such situations. Another example is the Isomap algorithm [13], which, adopting a graph-based approach, computes approximations of the geodesic distances on the manifold and uses them for producing lower dimensional representations of data. One problem that may arise in this process is that of topological instability - small perturbations on data points or minor changes in parameter 
values may result in large changes in the constructed approximate distances; cf. [2]. In this paper we present algorithms for distance approximation that are more robust against issues of topological instability. We study the performance of the methods on the popular swiss roll data set and discuss the influence of different choices of parameter values on the results. We find that our proposed methods are substantially more robust than the Isomap algorithm while retaining good performance on a global scale.

\section{Manifold Learning}

Let $\mathcal{X} \subset \mathbb{R}^{n}$ be a smooth manifold with intrinsic dimension $p \leq n$, and suppose that there exists a coordinate space $\mathcal{Y} \subset \mathbb{R}^{p}$ and a smooth, bijective mapping $\Phi: \mathcal{Y} \longrightarrow \mathcal{X}$. Assume that we are given data points $\hat{\mathcal{X}}_{m}=\left\{x_{1}, x_{2}, \ldots, x_{m}\right\} \subset$ $\mathcal{X}$, referred to as input coordinates, to which there correspond embedding coordinates, $\hat{\mathcal{Y}}_{m}=\left\{y_{1}, \ldots, y_{m}\right\}$ such that $\Phi\left(y_{j}\right)=x_{j}, j=1, \ldots, m$. The task of manifold learning is to, given $\hat{\mathcal{X}}_{m}$, and under suitable constraints, estimate properties of $\Phi$ and $\hat{\mathcal{Y}}_{m}$, or, more specifically, to find a mapping $M: \hat{\mathcal{X}}_{m} \longrightarrow \hat{\mathcal{Z}}_{m}$ from the input coordinates to the reconstructed embedding coordinates $\hat{\mathcal{Z}}_{m}=\left\{z_{1}, \ldots, z_{m}\right\}$, such that $\hat{\mathcal{Z}}_{m}$ and $\hat{\mathcal{Y}}_{m}$ are similar.

If $\Phi$ is a linear isometric embedding, the standard methods of PCA and classical MDS are useful manifold learning tools. For more general mappings, other techniques are required. In [13] the Isomap algorithm is introduced, treating the case where $\Phi$ is an isometry. Subsequently, C-Isomap [7] was proposed for conformal mappings.

Given a metric $d_{\mathbb{R}^{n}}$ on $\mathbb{R}^{n}$, Isomap calculates approximations of the geodesic distances $d_{\mathcal{X}}{ }^{1}$ on $\mathcal{X}$. An adjacency graph is constructed by connecting neighboring data points, where two points $x_{i}$ and $x_{j}$ are neighbors if either, given an $\epsilon>0, d_{\mathbb{R}^{n}}\left(x_{j}, x_{k}\right)<\epsilon$, or if, given an integer $K$, either one of $x_{j}$ and $x_{k}$, has the other among its $K$ closest points. As a next step, approximations $d_{\mathrm{ISO}}\left(x_{j}, x_{k}\right)$ of the geodesic distances $d_{\mathcal{X}}\left(x_{j}, x_{k}\right)$ on $\mathcal{X}$ are calculated by finding the shortest graph path between $x_{j}$ and $x_{k}$. If the data point density is high enough, $d_{\text {ISO }}$ approximates $d_{\mathcal{X}}$ well. Since $\Phi$ is an isometry, $d_{\text {ISO }}$ also approximates the Euclidean distances $d_{\mathcal{Y}}\left(y_{j}, y_{k}\right)$ in $\mathcal{Y}$. This motivates application of classical MDS to $d_{\mathrm{ISO}}\left(x_{j}, x_{k}\right)$, yielding lower dimensional representations of data.

The performance of the Isomap algorithm depends on the density of data points; the curvature of the manifold $\mathcal{X}$; the amount of noise; and the value of the neighborhood parameter $(K$ or $\epsilon)$; cf. [4]. For improper parameter choices or in the presence of noise, shortcuts, not following the surface of the manifold, may appear in the graph, disturbing the ability of the algorithm to approximate geodesic distances; cf. Fig. 1. This effect is known as the problem of topological instability; cf. [2]. Another property of the Isomap algorithm is that it tends

\footnotetext{
${ }^{1}$ i.e., the distance travelled by an insect, taking the shortest path between two points on the manifold.
} 


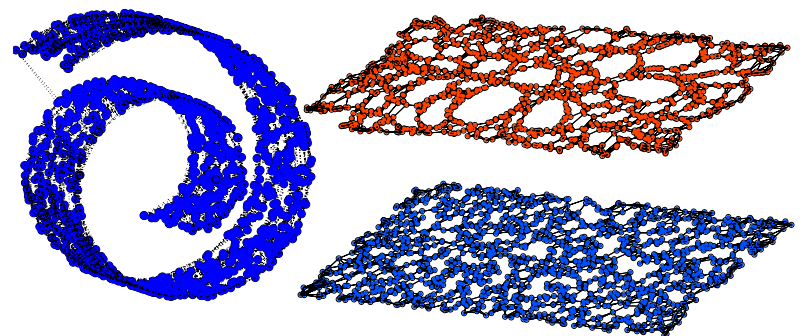

Fig. 1. To the left is shown 1500 uniformly distributed sample points $\left(\hat{\mathcal{X}}_{1500}\right)$ from a swiss roll manifold; the adjacency graph $(K=10)$ contains two shortcuts. To the right, the corresponding 1500 points in $\hat{\mathcal{Y}}_{1500}$, uniformly distributed in the plane (lower) and the corresponding Isomap projection (upper). The adjacency graph $(K=5)$ is drawn in the configurations. Random density fluctuations are amplified in the reconstruction

to cluster points in the resulting configurations. When the data point density on the manifold is finite, holes appear in the adjacency graph due to random fluctuations in local density, as illustrated in Fig. 1. For pairs of points on opposite sides of such holes the error term $\left|d_{\text {ISO }}-d_{\mathcal{X}}\right|$ will become larger and consequently cause the holes to grow in the resulting Isomap projection. As a consequence, the projection might exhibit structures which are in fact amplifications of random fluctuations in data density. Note, however, that the clustering effect is of local character, in contrast to the error caused by topological instability.

Isomap and C-Isomap are examples of global manifold learning methods, attempting to reconstruct the configuration in $\mathcal{Y}$ correctly on all scales. Local methods, on the other hand, attempt to create lower-dimensional representations that conserve similarities between nearby points only. Examples of local methods include Locally Linear Embedding [12], Laplacian Eigenmaps [3] and Hessian Eigenmaps [8]. Similarly to Isomap, the Laplacian Eigenmaps algorithm constructs an adjacency graph, connecting neighboring points, as an initial step. The graph edges are then given weights $w_{j k}=e^{-d_{\mathbb{R}^{n}}\left(x_{j}, x_{k}\right)^{2} / \sigma^{2}}$, for some coupling parameter $\sigma$. Let $W$ be the $m \times m$ matrix containing the elements $w_{j k}, B$ be a diagonal matrix defined by $B_{j j}=\sum_{k \neq j} w_{j k}$, and let $A=W-B$, i.e., the graph Laplacian. Briefly, the method of Laplacian Eigenmaps finds reconstruction embeddings by solving the generalized eigenvalue problem $A s=\lambda B s$.

\section{$3 \quad$ Circuit Models for Distance Measures}

Suppose that data points $x_{j} \in \mathcal{X}, j=1, \ldots, m$, are given, along with a distance metric $d_{\mathbb{R}^{n}}$. The aim of this paper is to construct methods to approximate the geodesic distance $d_{\mathcal{X}}\left(x_{j}, x_{k}\right)$ based only on the given data. Once good approximations are found, methods of MDS can be used to construct global methods for manifold learning. To this end, we employ models of electrical circuits, and use 
the dynamics of such to construct new distance approximations. This approach offers a framework for the construction and understanding of the models, which are reminiscent of the methods based on the graph Laplacian.

\subsection{A Passive Component Framework}

Associate each data point $x_{j}$ with a node $n_{j}$ in an electrical circuit. To each node, attach a capacitor with capacitance $c_{j}$ to earth. Neighboring ${ }^{2}$ node pairs $\left\{n_{j}, n_{p}\right\}$ are connected to each others by resistors $r_{j p}$, as in Fig. 2.a.

As a charge distribution is applied to the network, currents will move the charges until an equilibrium is reached, at which the voltages over the capacitors are all equal. The dynamic process of charging the capacitors will form the basis in our models for manifold learning, where we use charge times to construct new distance measures.

Denote the voltage over capacitor $c_{j}$ by $v_{j}(t)$, let $i_{j k}(t)$ denote the current from node $n_{j}$ to $n_{k}$, and let $i_{c_{j}}(t)$ denote the current from $n_{j}$ to $c_{j}$. According to the Kirchhoff current law,

$$
i_{c_{j}}(t)+\sum_{k=1, k \neq j}^{m} i_{j k}(t)=0 .
$$

The current between two nodes $n_{j}$ and $n_{k}$ is given by

$$
i_{j k}(t)=\frac{v_{j}(t)-v_{k}(t)}{r_{j k}},
$$

and for the current $i_{c_{j}}$ the relation

$$
c_{j} \frac{d v_{j}}{d t}=i_{c_{j}}(t)
$$

holds true. By applying (2) and (3) we can rewrite (1) into

$$
c_{j} \frac{d v_{j}}{d t}=\sum_{k=1, k \neq j}^{m} \frac{v_{k}(t)}{r_{j k}}-v_{j}(t) \sum_{k=1, k \neq j}^{m} \frac{1}{r_{j k}} .
$$

Let $V(t)$ be a column vector containing the elements $v_{j}(t), j=1, \ldots, m$. Then (4) can be expressed, in matrix form, as

$$
C \frac{d V(t)}{d t}=A V(t)
$$

where

$$
A(j, k)=\left\{\begin{array}{ll}
-\sum_{k \neq j} r_{j k}^{-1}, & \text { if } j=k ; \\
r_{j k}^{-1}, & \text { otherwise. }
\end{array},\right.
$$

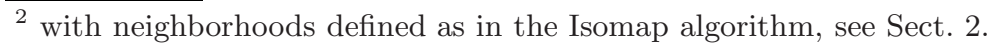


and $C$ is a diagonal matrix containing the capacities $c_{j}, j=1, \ldots m$. The solution to $(5)$ is then given by

$$
V(t)=e^{C^{-1} A t} V_{0},
$$

where $V_{0}$ is a column vector containing the initial voltages. It can be verified that $C^{-1} A$ is negative semi-definite.

Typically, we choose the resistances $r_{j k}$ to depend Gaussian on the distances $D_{j k}=d_{\mathbb{R}^{n}}\left(x_{j}, x_{k}\right)$, i.e., $r_{j k}=e^{d_{j k}^{2} / \sigma^{2}}$, for some parameter $\sigma$. The object $A$, with this choice of resistances, is the same as the graph Laplacian of Laplacian Eigenmaps; cf. Sect. 2.

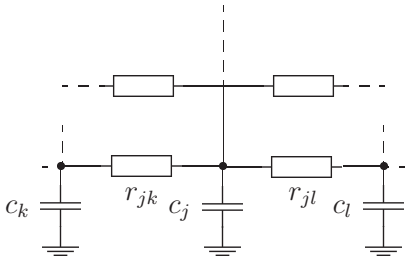

(2.a)

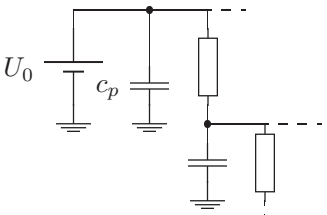

(2.b)

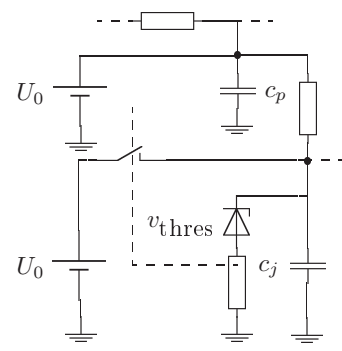

(2.c)

Fig. 2. Circuit models; a) The basic RC-circuit, b) The RC-circuit with constant voltage source, c) RC-unit with a voltage source connected through a zener diode switch

\subsection{The RC and RCZ Models}

Instead of the basic model with charges diffusing from an initial state, we adopt a model where one of the nodes, $n_{p}$, is attached to a constant voltage source, as illustrated in Fig. 2.b. The voltages at the other nodes will then monotonically increase and reach the battery voltage at infinity. By setting the capacity $c_{p}$ to infinity, we may use equation (5) to model the behavior of the circuit. The solution of the system is, analogously to (7), given by $V=e^{C^{\dagger} A t} V_{p}$, where $V_{p}(j)=\delta_{j p}$, and $C^{\dagger}(j, k)=c_{j}^{-1}$ if $j=k \neq p$ and 0 otherwise. There are several ways to define the distance between points based on the voltages $V$. A straightforward way is to use the time it takes for a node $n_{j}$ to reach a certain voltage level $0<v_{\text {thres }}<U_{0}$, with a typical value $v_{\text {thres }}=U_{0} / 2$. To assure symmetry we use the mean value of the time it takes to charge node $n_{j}$ given a constant voltage source at node $n_{p}$ and vice versa. These charge times need not fulfil the triangle inequality, why our dissimilarity measure is not necessarily a metric. This, however, does not disqualify its usefulness. For instance, the well known Mahalanobis measure is not metric; cf. [5]. In what follows, we will refer to the model above as the RC model, and denote the corresponding dissimilarity measure by $d_{\mathrm{RC}}$. More sophisticated dissimilarity measures could, e.g., be constructed based on statistical methods; cf. [9,11]. For our purposes, and in particular for what follows, the simpler variant is preferable. 
Consider a set of samples $\left\{x_{i}\right\}$ from a spiral $\mathcal{X}$, to which an outlier point is added, yielding a shortcut in the adjacency graph (Fig. 3.a.). Applying some global dimensionality reduction method, we would like the distances $d_{\circ}\left(x_{i}, x_{j}\right)$ estimated by the particular method to be closely related to the geodesic distance $d_{\mathcal{X}}\left(x_{i}, x_{j}\right)$ along the spiral. Ideally, there should be a linear relation, $d_{\circ}=a d_{\mathcal{X}}$. A more realistic criteria, though, would be to require a monotonically increasing relation $d_{\circ}=f\left(d_{\mathcal{X}}\right)$. Panels b-d of Fig. 3 display distances from the outmost point in the spiral, computed using different methods, plotted against the true geodesic distances. Disturbed by the graph shortcut, Isomap fails to compute distances that relate monotonically to $d_{\mathcal{X}}$ (Fig. 3.b). The RC method, on the other hand, succeeds with this despite the the shortcut (Fig. 3.c). However a shortcut effect still remains, and moreover, the relation between $d_{R C}$ and $d_{\mathcal{X}}$ deviates from a linear one. Points close to the source node are charged at a higher rate and during the time it takes for faraway points to reach threshold potential some charge will have trickled through the shortcut, thus explaining the observed shortcut influence. To avoid this, we introduce a model in which the nodes are charged through a moving front. In this model we connect nodes directly to the voltage source once the corresponding voltage reaches the threshold level $v_{\text {thres. }}$. At this instance, we say that the node reaches on-state, and we use the time is takes for the nodes to reach on-states as distance measure. In this way, nodes neighboring the front are charged directly by their neighbors, in contrast to being charged indirectly (through points in between) from the original source point. Electronically, we implement the moving front model by replacing the basic RC-unit with a slightly more sophisticated one; cf. Fig. 2.c. Each node is now equipped with a zener diode and a current controlled switch. As the voltage over the capacitor reaches $v_{\text {thres }}$, the zener diode moves into a conductive phase, turning the switch on, and the node enters the on-state. We refer to this model as the RCZ model, and denote the corresponding dissimilarity $d_{\mathrm{RCZ}}$.

The purpose of the RCZ model is to make distances more uniformly distributed, since points far from the original source node sooner will reach onstate. Indeed, applying the RCZ model on the spiral data results in distances scaling linearly with $d_{\mathcal{X}}$ showing just a minor influence from the shortcut (Fig. 3.d). For details on the numerical implementation of the RCZ model, see [1].

\section{Results and Discussion}

When both the coordinate space $\mathcal{Y}$ and the input space $\mathcal{X}$ are known (or, specifically, $\hat{\mathcal{Y}}_{m}$ and $\hat{\mathcal{X}}_{m}$ are given), we may evaluate the performance of dimensionality reduction methods by comparing the reconstructed configurations $\hat{\mathcal{Z}}_{m}$ with $\hat{\mathcal{Y}}_{m}$. A reasonable requirement on any algorithm is that it performs well on affine subspaces. One way of verifying this requirement, is to apply the method using the embedding coordinates, $\hat{\mathcal{Y}}_{m}$, as input. If $\Phi: \mathcal{Y} \longrightarrow \mathcal{X}$ is an isometry, the results should be identical when applying the algorithm to the real input coordinates $\hat{\mathcal{X}}_{m}$ as when applying it to $\hat{\mathcal{Y}}_{m}$. Thus, the performance evaluation can 


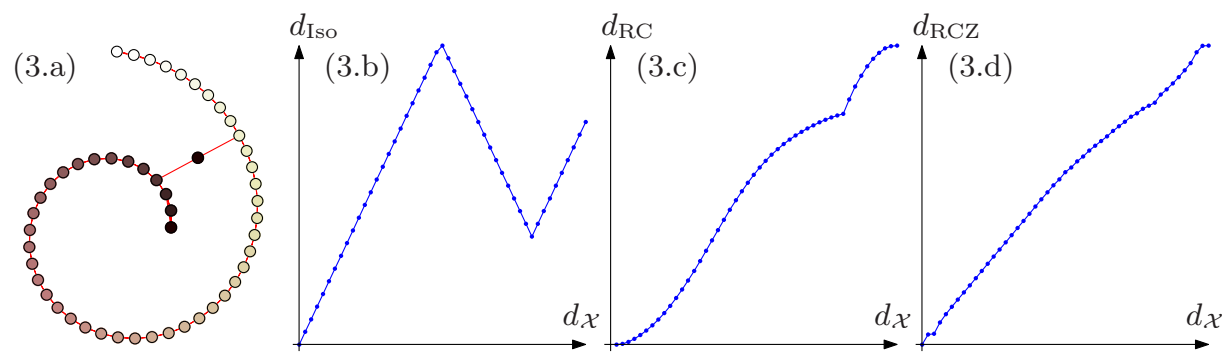

Fig. 3. Panel a shows perturbed spiral data. The remaining plots show the distances from the upper endpoint to the other points. Isomap distances (3.b) are severely distorted, while the RC (3.c) and the RCZ (3.d) distances deviate less from the geodesic

be divided with respect to two criteria - viewed as an operator, the method should mimic the identity operator when applied to $\hat{\mathcal{Y}}_{m}$, and the results when applied to $\hat{\mathcal{X}}_{m}$ should not deviate too much from the results when applied to $\hat{\mathcal{Y}}_{m}$.

When comparing point configurations, only their actual shapes are interesting. Hence, we remove issues of scale, rotation, reflection and translation by fitting the configurations optimally (in a least square sense) to each other using such transformations. Subsequently, the root mean square (RMS) error is taken as a measure of similarity, $E_{\mathrm{RMS}}=\sqrt{\sum_{i=1}^{m}\left|z_{i}-y_{i}\right|^{2} / m}$. In this section, we consider a set of 2000 data points, randomly sampled from a swiss roll manifold (cf. Fig. 1). The swiss roll data set contains coordinates for points both in $\mathcal{Y} \subset \mathbb{R}^{2}$ and on $\mathcal{X} \subset \mathbb{R}^{3}$, and is therefore a suitable test set. The coordinate space of the swiss roll is a rectangle in the plane, so dimensionality reduction methods should ideally retrieve this structure. We study the performance of Isomap, Laplacian Eigenmaps, RC and RCZ applied to this data set.

First, we consider the case where the data points coincide with the embedding coordinates, i.e., when $\hat{\mathcal{X}}_{2000}=\hat{\mathcal{Y}}_{2000} \subset \mathbb{R}^{2}$. Concerning the parameters, we choose $K=14$, a value where all four methods work well, and we choose the $\sigma$-values optimally for the Laplacian Eigenmaps and RCZ methods: $\sigma_{\mathrm{LEM}}=\infty$, $\sigma_{\mathrm{RCZ}}=3 \bar{d}$, where $\bar{d}$ is the average length in the adjacency graph, and use $\sigma_{\mathrm{RC}}=\sigma_{\mathrm{RCZ}}$. The resulting configurations are fitted to the embedding coordinates using similarity transformations, as described above, and the RMS errors of the respective reconstructions are calculated. Figure 4.a shows the embedding coordinates configurations $\left(\hat{\mathcal{Y}}_{2000}\right)$ by green/lighter points with the fitted reconstructions $\left(\hat{\mathcal{Z}}_{2000}\right)$ represented by blue/darker points, and with dotted (red) lines connecting the corresponding points in the configurations. On a global scale, Isomap and RCZ perform well while Laplacian Eigenmaps and RC suffer from some systematic errors. The $\mathrm{RC}$ reconstruction has an incorrect scale relation between the axes while Laplacian Eigenmaps produces a skewed reconstruction. Considering the local error structures, a slight clustering effect can be noticed in the Isomap reconstruction, while this effect is stronger in the other recon- 


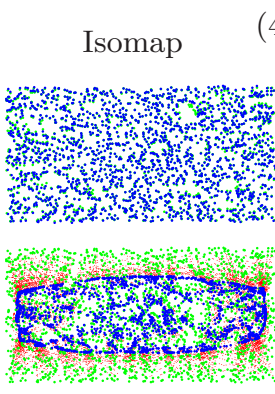

$\mathrm{RC}$ (4.a)

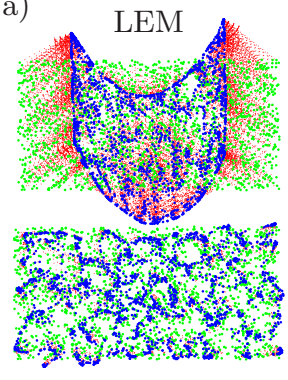

$\mathrm{RCZ}$



$\mathrm{RC}$

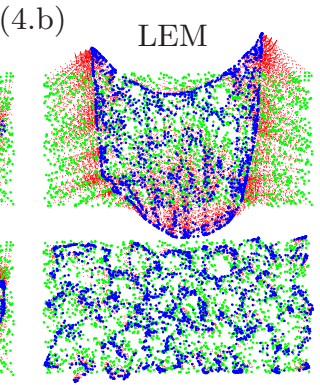

$\mathrm{RCZ}$

Fig. 4. Reconstructions with error structure: (a) for the planar case where the input coordinates and the embedding coordinates coincide; and (b) for the swiss roll case where the input coordinates are sampled from the swiss roll

structions. Clearly, Isomap produces the best reconstruction as also confirmed; $E_{\mathrm{RMS}}^{\mathrm{Iso}}=0.34, E_{\mathrm{RMS}}^{\mathrm{LEM}}=14, E_{\mathrm{RMS}}^{\mathrm{RC}}=6.8$, and $E_{\mathrm{RMS}}^{\mathrm{RCZ}}=3.0$.

Laplacian Eigenmaps does not aim for globally correct reconstructions, hence the global distortion is not surprising here. The RC global error is less severe and parts of it is presumably due to the nonlinear relation between geodesic and approximated distances, as discussed in Sect. 3. The punishment of long distances with a higher resistance in the $\mathrm{RC}$ methods leads to the increased sensitivity to local clustering errors. Since Laplacian Eigenmaps and the RC methods rely on similar equations, Laplacian Eigenmaps also exhibits these effects.

Next, we consider the point configuration $\hat{\mathcal{X}}_{2000}$ on the swiss roll manifold. Reconstructed embedding coordinates $\hat{\mathcal{Z}}_{2000}$ are computed using input coordinates $\hat{\mathcal{X}}_{2000} \subset \mathbb{R}^{3}$ on the swiss roll, and compared with $\hat{\mathcal{Y}}_{2000}$ (Fig. 4.b). The largest difference compared to the previous analysis occurs in the Isomap reconstruction where the two ends of the rectangle have become connected. For the other three methods, however, the differences are small. In other words, the Laplacian Eigenmaps, RC and RCZ reconstructions are reasonably invariant under $\Phi$ as required, while the Isomap reconstruction is not. The RMS errors are in this case $E_{\mathrm{RMS}}^{\mathrm{Iso}}=13, E_{\mathrm{RMS}}^{\mathrm{LEM}}=13, E_{\mathrm{RMS}}^{\mathrm{RC}}=6.4$, and $E_{\mathrm{RMS}}^{\mathrm{RCZ}}=3.0$, respectively.

Obviously, the results depend on the parameter values. For example, with $K=10$, no shortcuts appear in the adjacency graph on the swiss roll, and Isomap correctly reconstructs the rectangular configuration. In order to thoroughly investigate the method performances over a range of parameter values, we apply the algorithms using various $\sigma \in[\bar{d}, 4 \bar{d}]$ and $K=7, \ldots, 25$. Figure 5.a displays the approximation error for RCZ applied to the swiss roll (transparent surface), and the coordinate space rectangle (wire-frame mesh). Further, the difference between these two error matrices is shown by the lower surface plot, giving an idea of the fraction of error stemming from the geometrical change. The coordinate space error has a local minima around $K=14, \sigma=3 \bar{d}$. The difference between the two errors behaves more or less monotonic with increased 
$K$ and $\sigma$. Figure 5 .b shows the error $E_{\mathrm{RMS}}^{\mathrm{Iso}}$ of the Isomap reconstruction over different $K$ for the rectangle and the swiss roll respectively. A sharp increase in error for the swiss roll appears at $K=12$, where shortcuts first appear in the graph.

The error for the rectangle configuration is highest at low $K$ and low $\sigma$, where the local clustering effect is strongest - a low $K$ gives a higher probability of holes in the adjacency graph, while a low $\sigma$ gives a stronger punishment of long distances. At intermediate parameter values, the error is low, while it increases at higher $K$ and $\sigma$. This slightly surprising behavior might be partly explained as an edge effect - at the edges the density of graph edges will be higher, causing a tendency of constricting points along the rectangle edge. This effect grows with $K$ and $\sigma$. The fact that the residual error between the rectangle and the swiss roll grows with $K$ and $\sigma$ is explained by the increasing risk of shortcuts at larger $K$ and the decreasing capability of down-weighting them at larger $\sigma$.
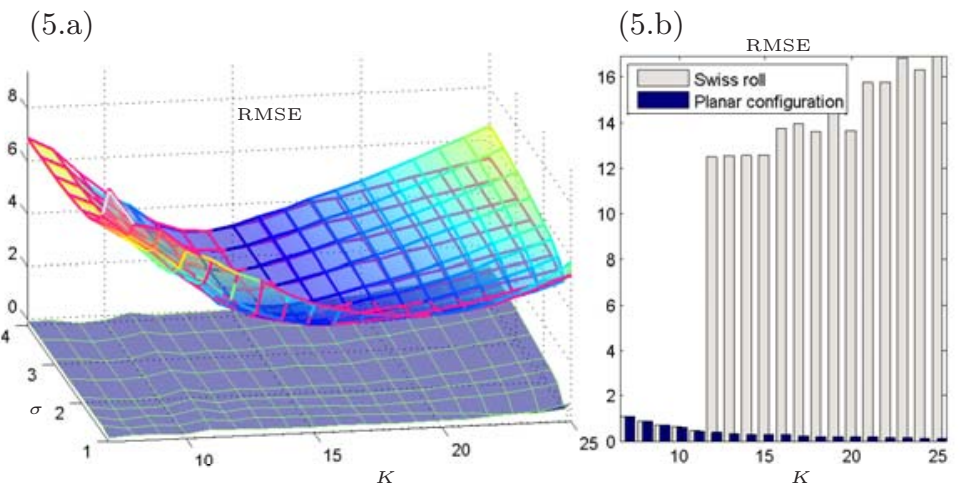

Fig. 5. a)RCZ: RMS errors for the planar and swiss roll configurations, illustrated by the colored surface and red wire-frame, respectively, with their difference, displayed by the lower surface; b) Isomap: RMS errors for the planar and swiss roll configurations

The results from the swiss roll data set illustrate that the Isomap algorithm is the most accurate, both on local and global scales - when it works, that is. Due to topological instability, it is less robust than the other methods. Being a local method, Laplacian Eigenmaps is more stable than Isomap but does not control the global correctness. Furthermore, it suffers from a larger local clustering error - a problem shared with the RC and RCZ methods. Because of their similarity with Laplacian Eigenmaps, we may view the RC methods as being akin to Laplacian Eigenmaps with global control added. Compared to Isomap we may regard the RC methods as more robust relatives who pay for the increased robustness with a larger local error. 


\section{Conclusions}

The present work addresses an alternative way of computing distances to avoid the problem of topological instability which the Isomap algorithm suffers from. These distances are obtained from solutions of ordinary differential equations, inspired by models of electric circuits. We demonstrate performance on typical data sets and discuss the relations between choice of parameter values and performance for the proposed and competing manifold learning methods, specifically under conditions of sparse or noisy data. The experiments show that the proposed methods may be seen as 'robustizations' of Isomap or 'globalizations' of Laplacian Eigenmaps.

\section{Acknowledgments}

The authors would like to thank Per Broberg and Magnus Fontes. The research of J.N. was partially supported by the Swedish Knowledge Foundation (KKstiftelsen), and AstraZeneca.

\section{References}

1. Andersson, F., Nilsson, J.: Circuit models for manifold learning. Tech. rep., Lund University (2005)

2. Balasubramanian, M., Schwartz, E. L., Tenenbaum, J. B., de Silva, V., Langford,J. C.: The Isomap algorithm and topological stability. Science 295 (2002)

3. Belkin, M., Niyogi, P.: Laplacian Eigenmaps for dimensionality reduction and data representation. Neural Computation 15 (2003) 1373-1396

4. Bernstein, M., de Silva, V., Langford, J. C., Tenenbaum, J. B.: Graph approximations to geodesics on embedded manifolds. Tech. rep., Stanford University (2000)

5. Chatfield, C., Collins, A. J.: Introduction to multivariate analysis. Chapman \& Hall, London (1980)

6. Cox, T. F., Cox, M. A. A.: Multidimensional scaling. Vol. 59 of Monographs on Statistics and Applied Probability. Chapman \& Hall, London (1994)

7. de Silva, V., Tenenbaum, J.: Global versus local methods in nonlinear dimensionality reduction. Neural Information Processing Systems 15 (2003), 705-712

8. Donoho, D., Grimes, C.: Hessian Eigenmaps: Locally linear embedding techniques for high-dimensional data. Proc. Natl. Acad. Sci. USA. 100(2003) 5591-5596

9. Ham, J., Lee, D. D., Mika, S., Schölkopf, B.: A kernel view of the dimensionality reduction of manifolds. In: ICML '04: Twenty-first international conference on Machine learning. ACM Press (2004)

10. Jolliffe, I. T.: Principal component analysis. Springer-Verlag, New York (2002)

11. Lafon, S.: Diffusion maps and geometric harmonics. Doctorate thesis, Yale University (2004)

12. Roweis, S. T., Saul, L. K.: Nonlinear Dimensionality Reduction by Locally Linear Embedding. Science 290 (2000) 2323-2326

13. Tenenbaum, J., de Silva, V., Langford, J.: A global geometric framework for nonlinear dimensionality reduction. Science 290 (2000) 2319-2322 